Check for updates

Cite this: RSC Adv., 2017, 7, 56594

Received 18th September 2017 Accepted 7th December 2017

DOI: 10.1039/c7ra10375a

rsc.li/rsc-advances

\section{An injectable in situ lipid phase transition system for sustained delivery of dabigatran etexilate with low burst release $\uparrow$}

\begin{abstract}
Xuanmiao Zhang, ${ }^{\text {ab }}$ Mei Hu, ${ }^{c}$ Guangfei Wei, ${ }^{c}$ Mengdi Jia, ${ }^{c}$ Tao Gong ${ }^{c}$ and Ji Liu (D) *a
Controlling initial burst release remains one of the greatest challenges in developing in situ-forming parenteral depot formulations. Here an injectable lipid phase transition system (LPTS) was fabricated to provide sustained release of dabigatran exilate (DABE) with low burst release. LPTS was prepared in a low-viscosity injectable state by simple mixing of sorbitan monooleate and phospholipids with ethanol. After subcutaneous injection, the liquid LPTS underwent a phase transition in situ and formed a semisolid mesophase, creating a drug-release depot. The physicochemical properties of DABE-LPTS were investigated using rheological techniques. Release profiles in vitro indicated that DABE-LPTS has suitable controlled release properties. Pharmacokinetic studies showed that DABE-LPTS significantly reduced the initial burst release after subcutaneous injection in rats. Pharmacodynamic studies showed that single administration of DABE-LPTS exerted anticoagulant effects in rats lasting 1 week. DABE-LPTS showed good biocompatibility in vitro and in vivo. Thus, the LPTS-based depot system shows promise for sustained DABE delivery with low burst release.
\end{abstract}

\section{Introduction}

Dabigatran exilate (DABE) is a novel oral reversible anticoagulant that offers potential advantages over existing anticoagulants such as warfarin for prophylaxis and treatment of thromboembolic disease. ${ }^{\mathbf{1 , 2}}$ By inhibiting thrombin directly, DABE prevents the conversion of fibrinogen into fibrin and thus prevents thrombus formation. ${ }^{\mathbf{1 , 2}}$ Given its low potential for drug-drug interactions, DABE shows great promise in anticoagulation therapy; in fact, it may change current practices in anticoagulation. . $^{3,4}$

DABE is administered orally in capsules. ${ }^{5}$ Despite its commercial success, this formulation of DABE is associated with bioavailability below $7 \%,{ }^{6}$ which can result in nonproportional and highly variable increases in drug plasma concentrations. ${ }^{7-9}$ Since DABE efficacy is strong, high interindividual variability in DABE concentrations can lead to an unacceptably high risk of bleeding. ${ }^{\mathbf{1 0 - 1 2}}$ The current oral formulation must be taken daily, which makes it less effective in patients who show poor compliance. This is a particular

${ }^{a}$ Department of Biochemistry and Molecular Biology, West China School of Basic and Forensic Medicine, Sichuan University, Chengdu 610041, China.E-mail: liuji6103@ scu.edu.cn

${ }^{b}$ Haisco Pharmaceutical Group Co. Ltd, Chengdu 611130, China

${ }^{c}$ Key Laboratory of Drug Targeting and Drug Delivery Systems, Ministry of Education, West China School of Pharmacy, Sichuan University, Chengdu 610041, China

$\dagger$ Electronic supplementary information (ESI) available. See DOI: 10.1039/c7ra10375a concern for patients who require long-term antithrombotic therapy, in whom accidental withdrawal of DABE may increase the risk of myocardial infarction and stroke. ${ }^{\mathbf{1 0 - 1 2}}$ In addition, the oral formulation is unsuitable for patients with dysphagia.

The ideal DABE delivery system would avoid the disadvantages of the current oral formulation and maintain plasma concentrations of the drug for a long time after a single administration. Sustained release injection could meet these requirements, but it would cause an initial burst release leading to high plasma concentration of DABE, which may increase risk of bleeding. ${ }^{11,12}$ This may help explain why, to the best of our knowledge, DABE delivery by sustained release injection has not been reported.

Such release may become possible using an injectable lipid phase transition system (LPTS). ${ }^{13-18}$ After subcutaneous injection, the aqueous body fluids penetrate into the LPTS, which leads to phase transition and precipitation of the lipid matrix, forming a depot at the injection site. ${ }^{13-16}$ LPTS can incorporate and control release of drugs of varying size and polar characteristics, and the lipids in LPTS are biodegradable. ${ }^{13-16}$ The purpose of the present study was to develop a DABE-loaded LPTS system to provide sustained release with low burst release. This study appears to be the first report of sustainedrelease DABE injection. The rheological behavior and in vitro release of DABE-loaded LPTS were evaluated. Subsequently, the pharmacokinetic and pharmacodynamic properties as well as toxicity of DABE-loaded LPTS after subcutaneous injection were investigated in animals. 


\section{Materials and methods}

\subsection{Materials, cells and animals}

DABE was obtained from JianFeng Chemical Company (Guangzhou, China). Purified yolk lecithin (Lipoid E80) was purchased from Lipoid (Ludwigshafen, Germany). Sorbitan monooleate (SMO) was obtained from Yuxiang Chemical (Guangzhou, China). Enoxaparin sodium was obtained from Hangzhou Jiuyuan Gene Engineering Co., Ltd. (Hangzhou, China). Platinum wires and pentobarbital sodium were obtained from Seebio Biotech Co., Ltd. (Shanghai, China). Pierce $^{\mathrm{TM}}$ Coomassie Plus (Bradford) Assay Kit was obtained from Thermo Fisher Scientific (USA). Other chemicals and reagents were of analytical grade and obtained commercially. CRL-1213 rat dermal fibroblasts were obtained from the American Type Culture Collection (USA). Sprague-Dawley rats were purchased from Dashuo Biotechnology (China). All animal procedures were performed in accordance with the Guidelines for Care and Use of Laboratory Animals of Sichuan University and approved by the Animal Experiment Administrative Committee of Sichuan University.

\subsection{Preparation of dabigatran exilate-loaded lipid phase transition system (DABE-LPTS)}

Lipoid E80 (500 mg) and SMO (400 mg) were mixed with $100 \mathrm{mg}$ of ethanol to form lipid matrix. Then, $20 \mathrm{mg}$ of DABE was added to the lipid matrix in a flask. The resultant mixture was stirred at $40{ }^{\circ} \mathrm{C}$ for $20 \mathrm{~min}$ to form DABE-LPTS.

\subsection{Rheological study}

The rheological behaviors of DABE-LPTS before and after gelation were determined at $25{ }^{\circ} \mathrm{C}$ using a Haake Mars III Rheometer (Thermo Scientific, USA) equipped with a parallelgeometry plate. In the case of DABE-LPTS in the sol state, the samples were directly subjected to viscosity measurement. In the case of DABE-LPTS in the gel state, a dialysis method was used to accomplish gel transition in vitro. DABE-LPTS solution was placed in the dialysis bag with a molecular weight cut-off of $5000 \mathrm{Da}$ and immersed in phosphate-buffered saline (PBS) at pH 7.4 at $37{ }^{\circ} \mathrm{C}$. After gelation, the DABE-LPTS was obtained and subjected to rheometry analysis.

First, a test was performed by measuring the viscosity as a function of shear rate. Second, the linear viscoelastic region (LVR) was determined in the amplitude sweep test at a constant frequency of $1.0 \mathrm{~Hz}$. Analysis of the LVR provided information about the appropriate strain required in the oscillation frequency sweep test. The oscillation frequency sweep test was then carried out by measuring the storage modulus $G^{\prime}$ and loss modulus $G^{\prime \prime}$ as a function of frequency ranging $0.01-100 \mathrm{~Hz}$ at a constant strain in LVR. This test was carried out to investigate sample behavior under constant strain and changing frequency.

\subsection{Water diffusion test}

To investigate the transition from the oil phase to the gel-like mesophase after DABE-LPTS came into contact with aqueous solution, $0.3 \mathrm{~mL}$ of DABE-LPTS was incubated in $10 \mathrm{~mL}$ of PBS ( $\mathrm{pH} 7.4)$ at room temperature. The water content of the DABELPTS was measured by Karl Fischer titration (787 KF Titrino, Metrohm, Switzerland) after incubation for 1, 3, 12, 36, 72, 168 and $240 \mathrm{~h}$.

\subsection{In vitro drug release}

The in vitro release of DABE from DABE-LPTS was investigated using the dialysis method. Initially, $0.2 \mathrm{~mL}$ of DABE-LPTS was placed into dialysis bags with a molecular weight cut-off of $5000 \mathrm{Da}$. Then the dialysis bags were immersed in $50 \mathrm{~mL}$ of the release medium (PBS, pH 7.4, containing varying percentages of ethanol) and incubated at $37{ }^{\circ} \mathrm{C}$ with constant shaking at $120 \mathrm{rpm}$. At fixed time intervals, $1 \mathrm{~mL}$ of release medium was sampled and replaced with $1 \mathrm{~mL}$ of fresh medium. Then the concentrations of DABE in the samples were determined by LCMS/MS (Agilent $1200 \mathrm{HPLC}$ /Agilent 6410B MS).

The diamonsil C18 column $(50 \times 4.6 \mathrm{~mm}, 1.8 \mu \mathrm{m})$ was used for separation at $30{ }^{\circ} \mathrm{C}$. The mobile phase consisted of methanol and deionized water containing $0.1 \%$ formic acid $(25: 75, \mathrm{v} / \mathrm{v})$, and the flow rate was $0.4 \mathrm{~mL} \mathrm{~min}^{-1}$. The mass spectrometer was operated using an electrospray source configured to negative ion mode and nitrogen was used as the nebulizer gas. The quantification analysis was performed using multiple reaction monitoring involving the transitions between $\mathrm{m} / \mathrm{z} 472.2$ (parent ion) and $\mathrm{m} / \mathrm{z} 289.0$ (gas temperature: $350{ }^{\circ} \mathrm{C}$; nebulizer: $35 \mathrm{psi}$; capillary: $4000 \mathrm{~V})$.

\subsection{Pharmacokinetics study in rats}

The DABE-LPTS (80 $\mathrm{mg} \mathrm{kg}^{-1}$ ) was subcutaneously injected into the back of each rat (six animals per group). Control animals were subcutaneously injected with DABE solution (11.4 $\mathrm{mg} \mathrm{kg}^{-1}$ ) as a seventh dose of DABE-LPTS group, since DABE-LPTS was hypothesized to deliver DABE for approximately one week. Blood samples were collected at different times and centrifuged at $6000 \mathrm{rpm}$ for $5 \mathrm{~min}$. Then, $500 \mu \mathrm{L}$ of acetonitrile was added to $100 \mu \mathrm{L}$ of the plasma sample. The mixture was subjected to vortex-mixing for $10 \mathrm{~min}$ and centrifuged at $13500 \mathrm{rpm}$ for $10 \mathrm{~min}$. The supernatant was taken for further analysis. The prepared samples were analyzed by LCMS/MS (Agilent 1200 HPLC/Agilent 6410B MS).

\subsection{In vivo anticoagulation effect}

The anticoagulation effect of DABE-LPTS was analyzed by measuring activated partial thromboplastin time (aPTT) as described. ${ }^{19}$ Sprague-Dawley rats were randomly divided into four groups $(n=6)$ and treated with one of the following formulations via subcutaneous injection: (1) DABE-LPTS (80 mg kg-1), (2) DABE solution (11.4 mg kg ${ }^{-1}$ ), (3) blank LPTS, or (4) physiological saline. Blood samples were collected from the orbit of each rat before treatment and at pre-determined time intervals after treatment. Samples were mixed with $0.109 \mathrm{~mol} \mathrm{~L}^{-1}$ trisodium citrate $(9: 1, \mathrm{v} / \mathrm{v})$ for anticoagulation, then centrifuged at $4500 \mathrm{rpm}$ for $10 \mathrm{~min}$ to separate the plasma, which was stored at $-80{ }^{\circ} \mathrm{C}$ until assay. The aPTT was measured using commercially available kits (Union Biotech, Chengdu, 
China) according to the manufacturer's instructions. The anticoagulation effect was defined as longer aPTT after treatment than before.

\subsection{Antithrombotic efficacy of DABE-LPTS in a rat model of venous thrombosis}

Sprague-Dawley rats were randomly divided into five groups ( $n$ $=12$ ) and treated with one of the following formulations via subcutaneous injection: (1) physiological saline $\left(0.3 \mathrm{~mL} \mathrm{~kg}{ }^{-1}\right.$ per day for continuous 7 days), (2) DABE-LPTS (80 $\mathrm{mg} \mathrm{kg}^{-1}$ ), (3) DABE solution (11.4 $\mathrm{mg} \mathrm{kg}^{-1}$ ), (4) enoxaparin injection (400 IU per $\mathrm{kg}$ per day for continuous 7 days), or (5) enoxaparin injection (1200 IU per kg per day for continuous 7 days). At 3 and 7 days after injection of DABE-LPTS, thrombosis was induced in six rats from each group using a platinum wire as described. ${ }^{\mathbf{2 0 , 2 1}}$ Rats were anesthetized with pentobarbital sodium $\left(60 \mathrm{mg} \mathrm{kg}^{-1}\right.$, intraperitoneal injection), and the inferior vena cava was exposed through a midline incision. Thrombus was induced by insertion of a platinum wire ( $2 \mathrm{~cm}$ long) into the inferior vena cava of rats just caudal to the left renal vein, and the wire remained in place for $60 \mathrm{~min}$. Immediately before removing the thrombus, $1 \mathrm{~mL}$ of $1 \%$ glutaraldehyde was injected into the inferior vena cava to fix the thrombus in situ. The wire with the thrombus was immediately dissected free and washed with saline. The protein content of the thrombus was measured using the Pierce ${ }^{\mathrm{TM}}$ Coomassie Plus (Bradford) Assay Kit (Thermo Scientific, USA) according to the manufacturer's instructions. Bovine serum albumin was used as a standard.

\subsection{Cytotoxicity assay}

The Cell Counting Kit-8 assay was used to evaluate the cytotoxicity of DABE-LPTS. Initially, $0.5 \mathrm{~mL}$ of DABE-LPTS was added to $5 \mathrm{~mL}$ of DMEM culture media (Hyclone, USA) with $10 \%$ fetal bovine serum (Hyclone, USA), and incubated at $37^{\circ} \mathrm{C}$ for $12 \mathrm{~h}$. After centrifugation, the supernatant of the mixture was taken as medium extract and diluted serially using DMEM with $10 \%$ fetal bovine serum to achieve final relative DABE-LPTS concentrations of 12.5, 25, 50 and 100\%. Fibroblasts (CRL-1213, ATCC, USA) were seeded in 96-well plates at $2 \times 10^{4}$ cells per well and cultured in DMEM containing $10 \%$ fetal bovine serum in a $\mathrm{CO}_{2}$ incubator $\left(37{ }^{\circ} \mathrm{C}, 5 \% \mathrm{CO}_{2}\right)$ for $24 \mathrm{~h}$. Then the medium was discarded and replaced with medium containing different relative concentrations of DABE-LPTS (100\%, 50\%, 25\%, 12.5\%), with $0.1 \%$ phenol as positive control. Cells were then incubated for 24 h. Afterward, cytotoxicity was determined using the Cell Counting Kit-8 (Beyotime Institute of Biotechnology, China) according to the manufacturer's instructions. Briefly, $10 \mu \mathrm{L}$ of CCK-8 solution containing WST-8 dye was added into each well and cells were incubated for another $1 \mathrm{~h}$ at $37^{\circ} \mathrm{C}$. WST-8 dye was reduced to soluble orange formazan by dehydrogenase in mitochondria during incubation. The optical density of soluble orange formazan at $450 \mathrm{~nm}$ was measured using a microplate reader. Cell viability (\%) was calculated according to the following equation: $\left(A_{\text {test }} / A_{\text {control }}\right) \times 100 \%$, where $A_{\text {test }}$ and $A_{\text {Control }}$ represent the absorbance of cells treated with, respectively, diluted medium extract or blank culture medium.

\subsection{Histological analysis}

Sprague-Dawley rats were subcutaneously injected with DABELPTS or physiological saline, then skin was collected from the injection site of each rat at 1, 14 and 21 days post-injection. In addition, rats were subcutaneously injected with DABE solution as positive control and the skin was collected from the injection site of each rat at 1 day post-injection. Skin was analyzed for pathology using hematoxylin-eosin (HE) staining.

\subsection{Statistical analysis}

Statistical comparisons were made using Student's $t$ test. $P<$ 0.05 was considered statistically different and $P<0.01$ was considered significantly different. All data reported are mean \pm standard deviation, unless otherwise noted.

\section{Results}

\subsection{Preparation and characterization of DABE-LPTS}

Here we prepared SMO and phospholipid solution in ethanol for the sustained delivery of DABE as an in situ-forming release depot. As shown in Fig. 1, phase transition occurred easily in DABE-LPTS in the presence of PBS. Initially, DABE-LPTS in the absence of a water phase was clear and transparent yellow in appearance, suggesting that the SMO, phospholipids and DABE formed a homogeneous mixture (Fig. 1A). LPTS transformed into a gel-like mesophase after coming into contact with PBS. As soon as LPTS was injected into PBS, it lost the flow properties of a liquid and changed into a spherical semi-solid form to minimize the contact surface with water, exhibiting a light yellow, semi-transparent appearance (Fig. 1B).

\subsection{Rheology}

Fig. 2A shows the evolution of shear viscosity against shear rate. DABE-LPTS before and after gelation both exhibited typical shear thinning behaviors, which implied that DABE-LPTS before and after gelation were non-Newtonian fluids. More information on the rheological behaviors of DABE-LPTS was obtained from dynamic strain sweep measurements and oscillatory shear frequency sweep measurements. Fig. 2B shows the relationship between moduli and strain in a dynamic strain

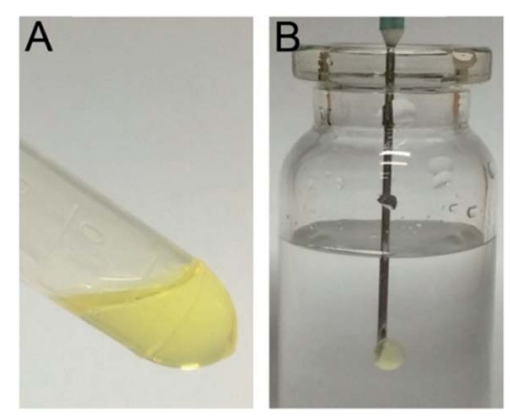

Fig. 1 The phase transition of DABE-LPTS. (A) Appearance of DABELPTS in the sol state before phase transition. (B) Appearance of DABELPTS in the gel state after injection into PBS. 
A

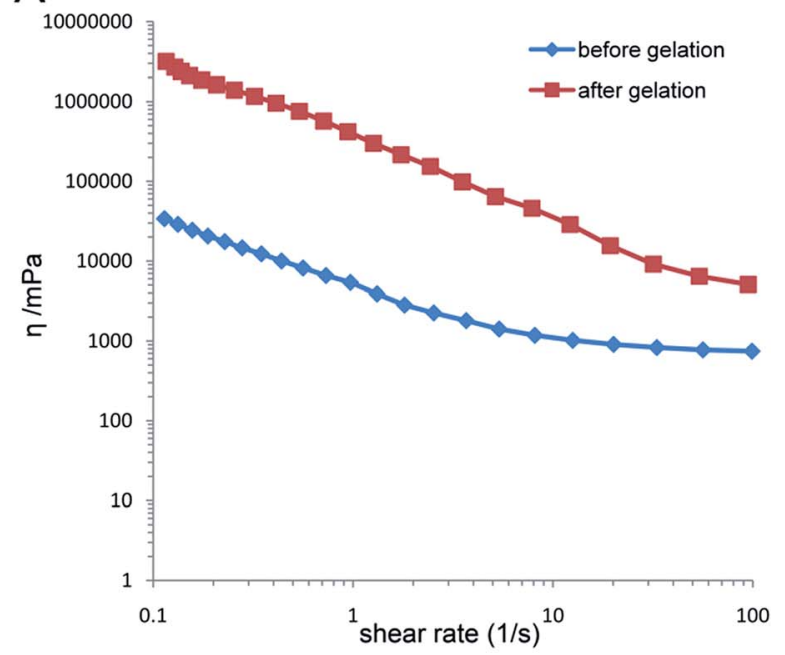

B

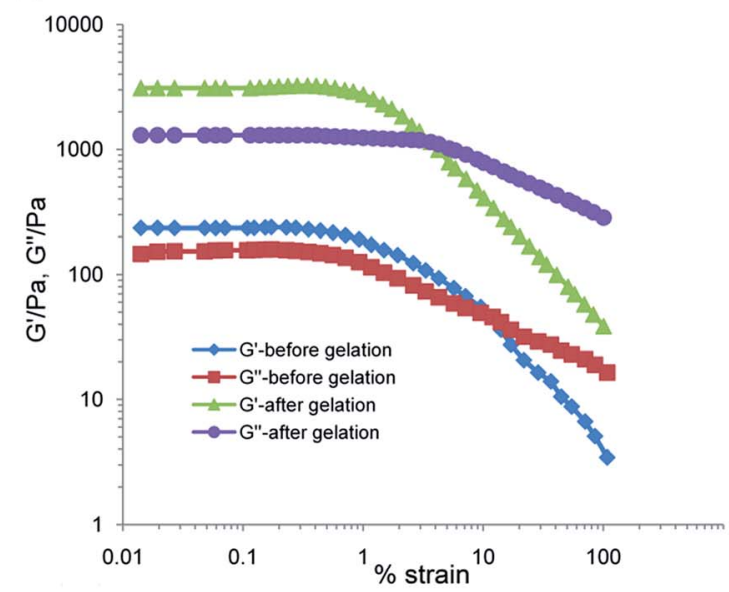

C

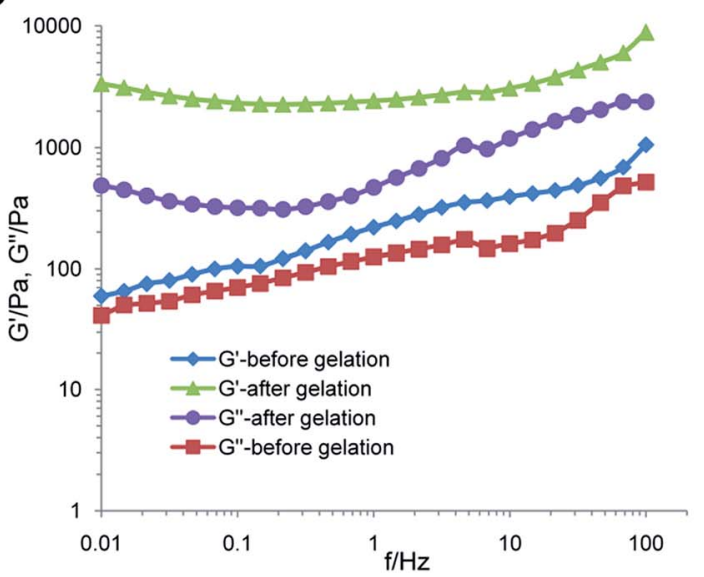

Fig. 2 Rheological behaviors of DABE-LPTS. (A) Steady shear viscosity as a function of shear rates for DABE-LPTS before and after gelation. (B) Relationship between moduli and strain for DABE-LPTS before and after gelation. (C) Moduli as a function of frequency for DABE-LPTS before and after gelation.

sweep experiment. Each sample showed a distinct LVR. The storage modulus $G^{\prime}$ and loss modulus $G^{\prime \prime}$ showed linear viscoelasticity before falling off steeply. $G^{\prime}$ was larger than $G^{\prime \prime}$ in the
LVR, indicating that elasticity was greater than viscidity. Based on the LVR acquired for each sample, we selected an appropriate strain value and performed oscillatory shear frequency sweep measurements, which allowed determination of the internal alteration in sample structure. The frequency dependence of $G^{\prime}$ and $G^{\prime \prime}$ for DABE-LPTS is shown in Fig. 2C. After gelation, the $G^{\prime}$ and $G^{\prime \prime}$ of DABE-LPTS increased. $G^{\prime}$ increased much faster than $G^{\prime \prime}$, indicating that DABE-LPTS became highly elastic during phase separation.

\subsection{Water diffusion test}

DABE-LPTS was incubated in PBS, and its water content was measured at different time points. Water content increased up to $23.6 \%$ at $168 \mathrm{~h}$ and remained constant thereafter (Fig. 3A), indicating that water content probably cannot exceed $24 \%$ for successful formation of a gel-like mesophase.

\subsection{In vitro drug release profile}

The release profiles of DABE from DABE-LPTS showed a significant delayed pattern under all conditions for over 2 days. The presence of ethanol in the release medium resulted in faster DABE release and higher cumulative release percentage. Negligible DABE was released from DABE-LPTS even after 2 days in PBS containing $10 \%(\mathrm{v} / \mathrm{v})$ ethanol, whereas nearly $40 \%$ of DABE was released from DABE-LPTS in PBS containing 30\%
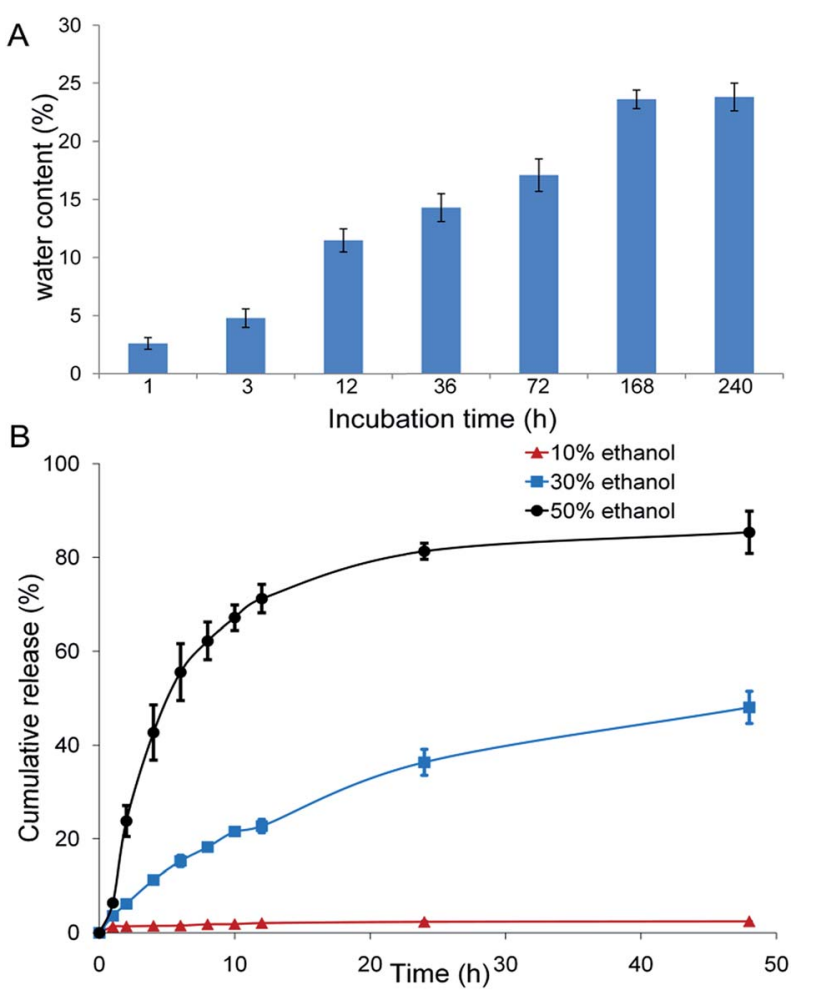

Fig. 3 Water content of DABE-LPTS and in vitro release of DABE. (A) Water content of DABE-LPTS after incubation in PBS ( $\mathrm{pH}$ 7.4). Data are mean $\pm \mathrm{SD}(n=3)$. (B) In vitro release profiles of DABE from DABELPTS in release media containing 10,30 or $50 \%$ of ethanol $(v / v)$ in PBS $(\mathrm{pH}$ 7.4). Data are mean $\pm \mathrm{SD}(n=3)$. 
Table 1 Fitting equations and correlation coefficients resulting from the application of different mathematical models to data on the release of DABE from DABE-LPTS in vitro in different release media

\begin{tabular}{lllll}
\hline Release media & First order kinetics & Higuchi & Weibull & Ritger-Peppas \\
\hline PBS containing & $\ln (1-Q)=-0.001 t-0.015$, & $Q=0.002 t^{1 / 2}-0.011$, & $\ln \ln [1 /(1-Q)]=-0.045 \ln (t)$ & $\ln Q=0.183 \ln t-4.401$, \\
10\% ethanol & $R^{2}=0.774$ & $R^{2}=0.91$ & $+1.484, R^{2}=0.926$ & $R^{2}=0.929$ \\
PBS containing & $\ln (1-Q)=-0.013 t-0.078$, & $Q=0.077 t^{1 / 2}-0.038$, & $\ln \ln [1 /(1-Q)]=-0.391 \ln (t)$ & $\ln Q=0.681 \ln t-3.193$, \\
$30 \%$ ethanol & $R^{2}=0.958$ & $R^{2}=0.993$ & $+1.294, R^{2}=0.983$ & $R^{2}=0.985$ \\
PBS containing & $\ln (1-Q)=-0.035 t-0.501$, & $Q=0.124 t^{1 / 2}+0.161$, & $\ln \ln [1 /(1-Q)]=-0.756 \ln (t)$ & $\ln Q=0.616 \ln t-2.018$, \\
$50 \%$ ethanol & $R^{2}=0.76$ & $R^{2}=0.751$ & $+0.885, R^{2}=0.988$ & $R^{2}=0.782$
\end{tabular}

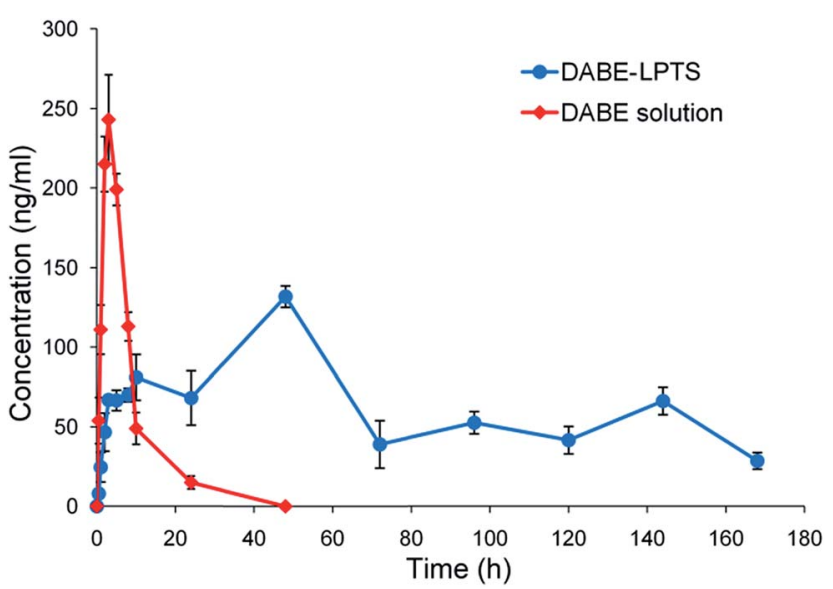

Fig. 4 Concentration-time profiles of DABE in plasma after subcutaneous injection of DABE-LPTS and DABE solution in rats. Data are mean $\pm \operatorname{SD}(n=6)$.

(v/v) ethanol (Fig. 3B). The kinetics of DABE release from DABELPTS was fitted to several classical mathematical models; the $R^{2}$ of most models was above 0.9 , with the Higuchi and Weibull model showing the best fit to the data (Table 1). These findings suggest that LPTS helps control and sustain the release of DABE.

\subsection{Pharmacokinetics in rats}

The pharmacokinetic properties of DABE-LPTS and DABE solution were assessed after a single subcutaneous injection in rats. Fig. 4 shows concentration $v s$. time profiles of DABE in plasma. DABE-LPTS showed lower $C_{\max }$ than the control, even though DABE-LPTS contained 7-fold more DABE.

Following the initial release phase, DABE-LPTS maintained a stable DABE concentration between 20 and $168 \mathrm{~h}$. In fact,
DABE release from DABE-LPTS lasted for several days. These results demonstrate that DABE-LPTS can release DABE in a sustained manner, with low initial burst release and stable control of DABE concentration. This implies that LPTS functions as a reservoir for controlled DABE release. The plasma kinetics of DABE solution and DABE-LPTS are shown in Table 2. DABE-LPTS can significantly lengthen DABE half-life compared with its half-life in DABE solution.

\subsection{In vivo anticoagulation effect of DABE-LPTS}

The coagulation marker aPTT is used to evaluate the ability of DABE to prolong clotting time. ${ }^{22}$ Animals that received physiological saline or blank LPTS showed aPTT near baseline (data not shown), suggesting that the biomaterials did not alter the drug's anticoagulant efficacy. The anticoagulation effect lasted fewer than $20 \mathrm{~h}$ after a single administration of DABE solution but 1 week after a single administration of DABE-LPTS (Fig. 5). Injection with DABE-LPTS was associated with increasing aPTT at initial time points, beyond which aPTT remained relatively constant and then decreased to baseline after 7 days.

\subsection{Antithrombotic effects of DABE-LPTS}

In the rat model of venous thrombosis, thrombus formation was induced on the surface of the platinum wire in the inferior vena cava. As shown in Fig. 6, no significant antithrombotic efficacy was observed on day 3 or 7 after injection with DABE solution, whereas significant thrombus inhibition was observed at the same time points after injection with DABE-LPTS. Next, the antithrombotic efficacy of DABE-LPTS was compared with that of enoxaparin, a classical antithrombotic agent that is effective with daily subcutaneous injection. ${ }^{33}$ As shown in Fig. 6, DABE-LPTS showed antithrombotic efficacy comparable to that of enoxaparin. This provides further evidence that DABE-LPTS supports sustained release of DABE.

Table 2 Pharmacokinetic parameters of DABE in plasma after subcutaneous administration of DABE solution or DABE-LPTS in rats. Values are mean $\pm \mathrm{SD}(n=6)$

\begin{tabular}{lccccc}
\hline Group & $T_{\max }(\mathrm{h})$ & $C_{\max }\left(\mathrm{ng} \mathrm{mL}^{-1}\right)$ & $t_{1 / 2}(\mathrm{~h})$ & $\mathrm{MRT}_{0-t}(\mathrm{~h})$ & $\mathrm{AUC}_{0-t}\left(\mathrm{ng} \mathrm{mL}^{-1} \mathrm{~h}^{-1}\right)$ \\
\hline DABE solution & $3.18 \pm 0.21$ & $243.86 \pm 31.47$ & $5.21 \pm 0.83$ & $7.86 \pm 1.14$ & $1966.75 \pm 473.82$ \\
DABE-LPTS & $48.21 \pm 3.56^{a}$ & $131.95 \pm 22.56^{a}$ & $96.14 \pm 8.72^{a}$ & $138.42 \pm 26.93^{a}$ & $10774.2 \pm 869.24^{a}$
\end{tabular}

${ }^{a} P<0.01$ vs. DABE solution. 


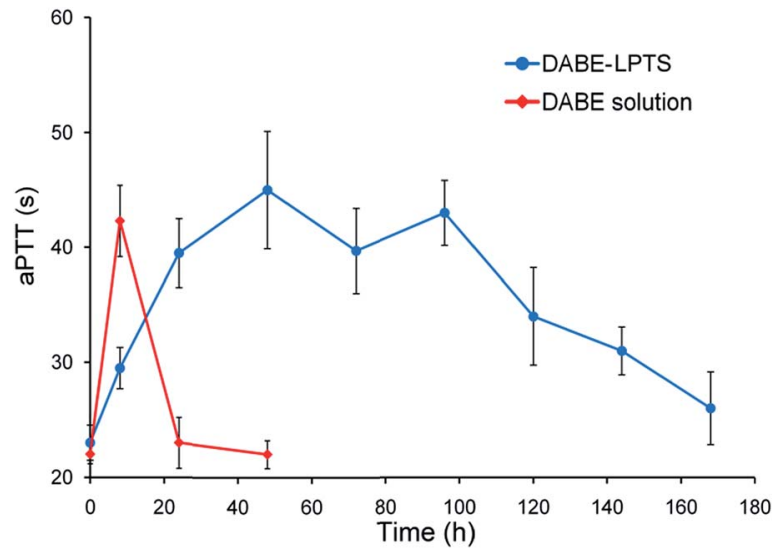

Fig. 5 Time course of aPTT after subcutaneous administration of DABE solution or DABE-LPTS in Sprague-Dawley rats. Data are mean $\pm \mathrm{SD}(n=6)$. No anticoagulation effects were observed in control animals treated with physiological saline or blank LPTS (data not shown).
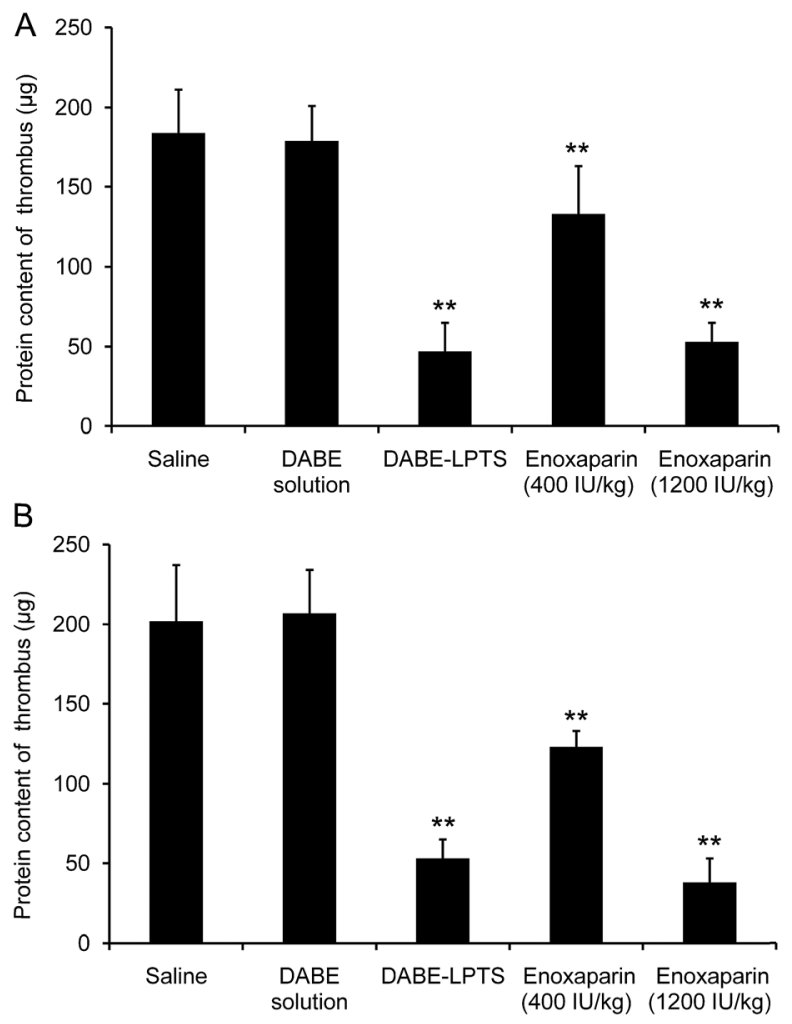

Fig. 6 Thrombus protein content in a rat model of platinum wireinduced venous thrombosis on day $3(A)$ and day 7 (B) after injection of DABE-LPTS. Data are mean \pm SD $(n=6)$. ${ }^{* *} P<0.01$ vs. saline.

\subsection{Preliminary safety evaluation}

In vitro cytotoxicity of DABE-LPTS is illustrated in Fig. 7. No significant differences were observed among cultures treated with various concentrations of DABE-LPTS, and over $90 \%$ of cells survived after $24 \mathrm{~h}$ treatment, in contrast to the positive control cells treated with $0.1 \%$ phenol. These results indicate

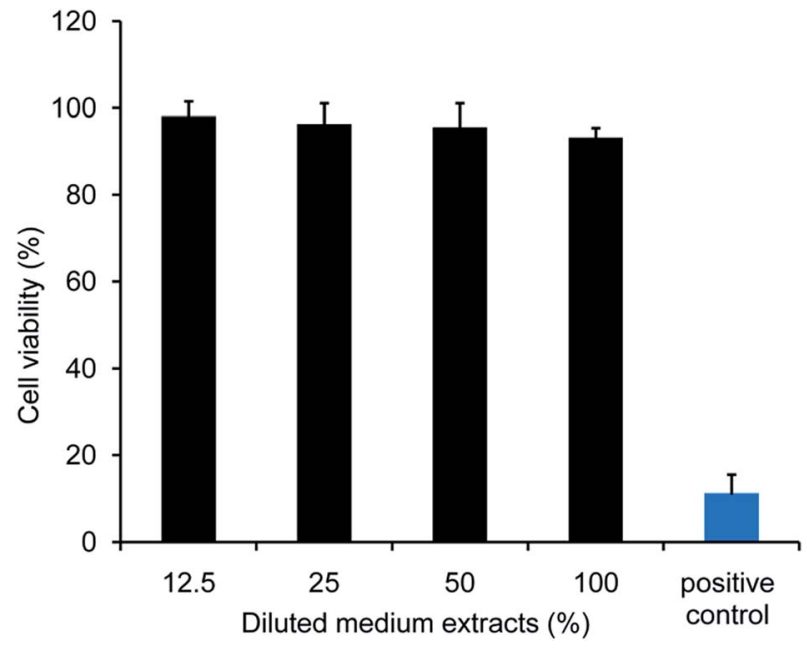

Fig. 7 Cytotoxicity of DABE-LPTS extracts of different concentrations on rat dermal fibroblasts. Unexposed cells served as a negative control, and cells exposed to $0.1 \%$ phenol served as a positive control. Data are mean $\pm \operatorname{SD}(n=6)$.

that DABE-LPTS did not induce significant cytotoxicity in vitro at the concentrations tested.

Next the biocompatibility of DABE-LPTS was assessed by examining local dermal irritation caused by a single subcutaneous injection of DABE-LPTS. Injection did not alter normal behavior or feeding of any animals. As shown in Fig. 8, DABELPTS did not induce significant pathological changes at the injection site during the experiment. These results demonstrate that DABE-LPTS was well tolerated in vivo.

\section{Discussions}

An LPTS based on SMO, phospholipid and ethanol was developed for the sustained delivery of DABE. In this system, SMO and phospholipid constitute the main components that modulate drug release. A low percentage of ethanol serves as water-miscible organic solvent to keep the LPTS in a lowviscosity injectable state before the phase transition. Preparing the LPTS is simple: the components need only be mixed with stirring, giving rise to a sol state before injection. After contact with aqueous fluids, the amphiphilic SMO and phospholipid in the LPTS realign and a mesophase forms spontaneously, creating a drug release depot at the injection site.

Despite extensive research with LPTS, no such system has been clinically approved for subcutaneous delivery. Key to clinical translation of such systems are the safety and parenteral acceptability of excipients used in the LPTS. ${ }^{23}$ Our system uses phospholipid and SMO, which show low toxicity and good biological tolerance. SMO is an injectable emulsifier that is already in use in various pharmaceutical formulations, unlike other amphiphilic lipids such as glycerol monooleate or glycerol monolinoleate. ${ }^{16}$

Key to commercialization of an injectable sustained release drug delivery system is the feasibility of industrial scale-up. The 


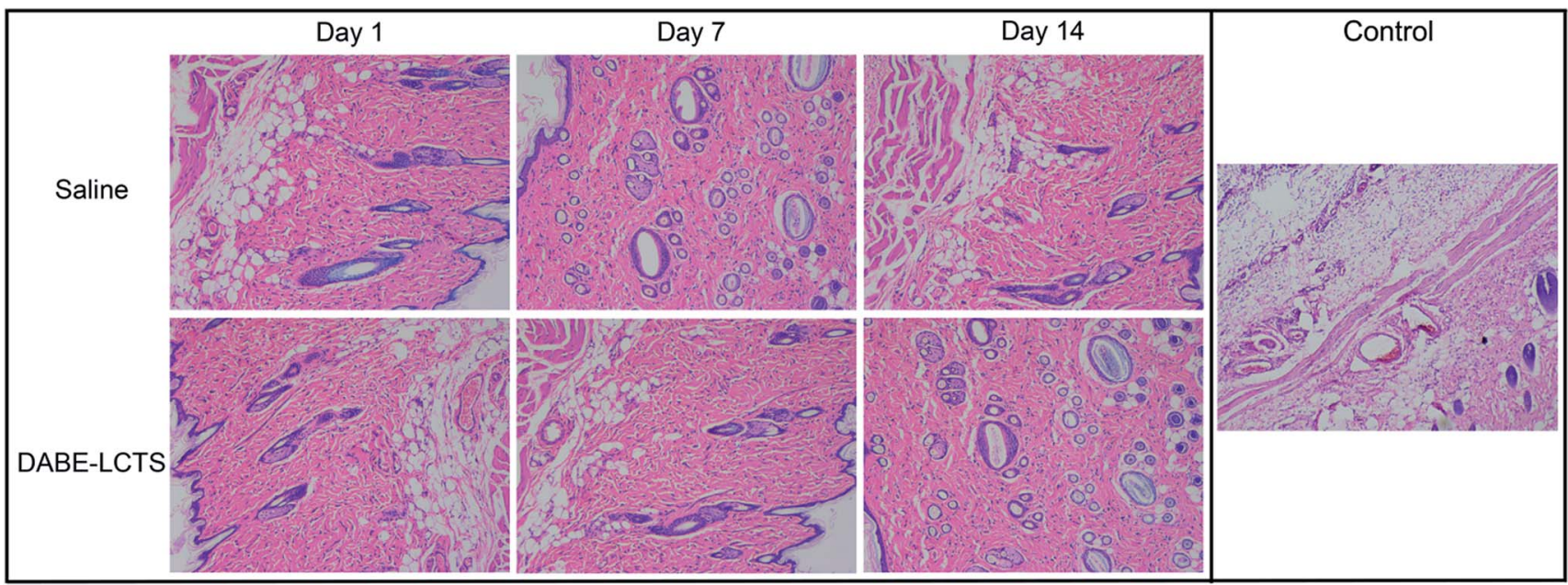

Fig. 8 Preliminary safety evaluation of LPTS formulations through hematoxylin-eosin staining of rat skin samples taken on days 1, 14 and 21 after subcutaneous injection of DABE-LPTS or physiological saline. Rats treated with DABE solution served as positive control (magnification, $100 \times$ ).

simple preparation of DABE-LPTS by mixing and stirring should lend well to scale-up, and the formulation can be filtersterilized without compromising phase behavior or sustained drug release.

Cumulative release of DABE in our in vitro experiments increased with ethanol concentration in the release medium, which may reflect the fact that both excipients, phospholipid and SMO, are soluble in ethanol. Thus, increasing ethanol content in the release medium may facilitate diffusion of phospholipid and SMO out of the gel matrix, accelerating matrix degradation and drug release.

A major challenge to developing delivery systems for sustained DABE release is the need to avoid an initial burst release that could increase risk of adverse events, particularly bleeding..$^{24,25}$ With an LPTS, the initial burst of drug release can occur during the lag period between injection and depot formation. ${ }^{26}$ Our DABE-LPTS showed a significantly reduced initial burst of DABE release, suggesting promise for clinical use.

Antithrombotic drugs, which include antiplatelet and anticoagulant agents, are widely used to prevent and treat venous thromboembolism as well as prevent stroke in patients with atrial fibrillation. ${ }^{27}$ Antithrombotic therapy has long been dominated by warfarin, clopidogrel, parenteral unfractionated heparin, and low-molecular-weight heparin. ${ }^{28}$ New treatment options include antiplatelet drugs (prasugrel, ticagrelor) and inhibitors that directly target factor IIa (dabigatran) or Xa (rivaroxaban, apixaban, edoxaban). ${ }^{27}$ The efficacy of all of these drugs is limited by inadequate pharmacokinetics ${ }^{29,30}$ including short half-life that necessitates daily administration. This makes drug efficacy strongly dependent on patient compliance, and it increases risk of bleeding. ${ }^{29}$ On the other hand, spacing the doses increases the risk of complications such as myocardial infarction and stroke. ${ }^{27}$ This highlights the need to develop drug delivery systems that can maintain antithrombotic activity high enough to avoid new clot formation but not high enough to trigger drug-related toxicity. ${ }^{29}$
In the present study, we provide a potentially superior alternative to various drug delivery systems that can improve the pharmacokinetic properties of antithrombotic drugs and prolong their pharmacological effects. ${ }^{31-33}$ Our DABE-LPTS showed good antithrombotic effects lasting 1 week after a single injection. This presents much less of a patient compliance problem than the current DABE formulation as an oral capsule, and it facilitates long-term antithrombotic therapy, such as for patients with cancer or with prosthetic heart valves. ${ }^{29}$

\section{Conclusions}

Our results suggest that LPTS can be used for sustained delivery of DABE with low burst release. The LPTS was formulated using phospholipid and SMO as core components and ethanol as solvent. Preliminary safety evaluation showed that LPTS did not cause significant pathology, such as inflammation at the injection site. DABE-LPTS showed suitable controlled drug release profiles in vitro and in vivo. Single administration of DABE-LPTS in rats led to anticoagulation effects lasting 1 week. Thus, LPTS-based depot technology represents a promising local drug delivery strategy for sustained release of DABE with long-term anticoagulation effects and low toxicity.

\section{Conflicts of interest}

There are no conflicts to declare.

\section{Acknowledgements}

This work was supported by the National Natural Science Foundation of China (81690261 and 81673359).

\section{References}

1 W. Ageno, J. Eikelboom and G. Y. Lip, Int. J. Cardiol., 2016, 220, 417-428. 
2 S. L. Greig and K. McKeage, Drugs, 2014, 74, 1785-1800.

3 T. Baglin, Br. J. Haematol., 2013, 163, 160-167.

4 R. Providencia, J. P. Albenque, S. Combes, A. Bouzeman,

B. Casteigt, N. Combes, K. Narayanan, E. Marijon and

S. Boveda, Heart, 2014, 100, 324-335.

5 M. Remko, J. Mol. Struct.: THEOCHEM, 2009, 916, 76-85.

6 S. J. Connolly, M. D. Ezekowitz, S. Yusuf, J. Eikelboom, J. Oldgren, A. Parekh, J. Pogue, P. A. Reilly, E. Themeles, J. Varrone, S. Wang, M. Alings, D. Xavier, J. Zhu, R. Diaz, B. S. Lewis, H. Darius, H. C. Diener, C. D. Joyner and L. Wallentin, N. Engl. J. Med., 2009, 361, 1139-1151.

7 I. Gouin-Thibault, X. Delavenne, A. Blanchard, V. Siguret, J. E. Salem, C. Narjoz, P. Gaussem, P. Beaune, C. FunckBrentano, M. Azizi, P. Mismetti and M. A. Loriot, J. Thromb. Haemostasis, 2017, 15, 273-283.

8 I. Y. Gong and R. B. Kim, Can. J. Cardiol., 2013, 29, S24-S33. 9 J. Douxfils, F. Mullier and J. M. Dogne, Expert Opin. Drug Saf., 2015, 14, 1283-1289.

10 N. S. Abraham, S. Singh, G. C. Alexander, H. Heien, L. R. Haas, W. Crown and N. D. Shah, BMJ, 2015, 350, h1857.

11 J. Douxfils, F. Buckinx, F. Mullier, V. Minet, V. Rabenda, J. Y. Reginster, P. Hainaut, O. Bruyere and J. M. Dogne, J. Am. Heart Assoc., 2014, 3, e000515.

12 B. J. Bloom, K. B. Filion, R. Atallah and M. J. Eisenberg, Am. J. Cardiol., 2014, 113, 1066-1074.

13 T. Zhang, Q. Peng, F. Y. San, J. W. Luo, M. X. Wang, W. Q. Wu, T. Gong and Z. R. Zhang, Biomaterials, 2015, 45, 1-9.

14 M. Wang, F. Shan, Y. Zou, X. Sun, Z. R. Zhang, Y. Fu and T. Gong, J. Controlled Release, 2016, 230, 45-56.

15 L. Han, J. Xue, L. Wang, K. Peng, Z. Zhang, T. Gong and X. Sun, Biomaterials, 2016, 105, 185-194.

16 M. H. Ki, J. L. Lim, J. Y. Ko, S. H. Park, J. E. Kim, H. J. Cho, E. S. Park and D. D. Kim, J. Controlled Release, 2014, 185, 62-70.

17 H. Li, T. Liu, Y. Zhu, Q. Fu, W. Wu, J. Deng, L. Lan and S. Shi, Acta Biomater., 2017, 58, 136-145.
18 D. Long, G. Tao, Z. Zhang, D. Rui and F. Yao, Acta Pharm. Sin. $B, 2016,6,329-335$.

19 M. Hu, J. Zhang, R. Ding, Y. Fu, T. Gong and Z. Zhang, Drug Dev. Ind. Pharm., 2017, 43, 687-697.

20 T. Furugohri, K. Isobe, Y. Honda, C. Kamisatomatsumoto, N. Sugiyama, T. Nagahara, Y. Morishima and T. Shibano, J. Thromb. Haemostasis, 2008, 6, 1542-1549.

21 Y. Morishima, Y. Honda, C. Kamisato, N. Tsuji, A. Kita, N. Edo and T. Shibano, Thromb. Res., 2012, 130, 514-519.

22 J. Stangier, Clin. Pharmacokinet., 2008, 47, 285-295.

23 S. Kempe and K. Mader, J. Controlled Release, 2012, 161, 668679.

24 M. N. Di Minno, P. Ambrosino, A. Di Minno, E. Tremoli and G. Di Minno, Ann. Med., 2017, 49, 329-342.

25 P. K. Bundhun, M. Z. Soogund, A. R. Teeluck, M. Pursun, A. Bhurtu and W. Q. Huang, BMC Cardiovasc. Disord., 2017, 17, 15.

26 R. R. Thakur, H. L. McMillan and D. S. Jones, J. Controlled Release, 2014, 176, 8-23.

27 J. L. Mega and T. Simon, Lancet, 2015, 386, 281-291.

28 Y. Iwatsuki, T. Sato, Y. Moritani, T. Shigenaga, M. Suzuki, T. Kawasaki, T. Funatsu and S. Kaku, Eur. J. Pharmacol., 2011, 673, 49-55.

29 C. F. Greineder, M. D. Howard, R. Carnemolla, D. B. Cines and V. R. Muzykantov, Blood, 2013, 122, 1565-1575.

30 E. Shantsila, Expert Opin. Drug Metab. Toxicol., 2013, 9, 13351347.

31 M. R. Matanović, P. A. Grabnar, D. Voinovich, S. Golob, M. B. Mijovski and I. Grabnar, Int. J. Pharm., 2015, 496, 583-592.

32 S. S. Ibrahim, R. Osman, A. Gas, N. D. Mortadaa and A. S. Geneidi, Int. J. Pharm., 2017, 526, 271-279.

33 C. Pazzini, P. D. Marcato, L. B. Prado, A. M. Alessio, N. F. Höehr, S. Montalvão, D. Paixão, N. Durán and J. M. Annichino-Bizzacchi, J. Nanosci. Nanotechnol., 2015, 15, 4837-4843. 